\title{
Análisis de las variables implicadas en la detección de la mentira
}

\author{
ALBERTO BECERRA \\ FLOR SÁNCHEZ \\ Universidad Autónoma de Madrid

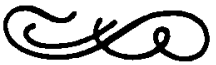

\begin{abstract}
Resumen
El trabajo que presentamos profundiza en el estudio de variables relevantes, para la detección de la mentira, tales como el tema, el tipo de mentira, y el sujeto emisor. Nuestro diseño de investigación establece una subdivisión de los mensajes falsos en deseables y no deseables y clasifica el contenido de los mensajes en emocional y no emocional, postulando que estas diferencias generan diferentes patrones de activación y expresión en los sujetos emisores, que serán detectados por los receptores de los mensajes.
\end{abstract}

\section{Abstract}

The aim of the research here reported is to study in depth the analysis of relevant variables in the detection of deception such as the topic, type of expression, and sender! Our design divides false expressions into two sub-types: adesirable (i.e., with a content that the sender would find it desirable to be true and "non-desirablex, and classified the content of messages into "emotional* and "no-emotional. Then, we hypothesized that these differences would generate different expressions and activation patterns in the sender that would then be detected by the receptors.

Agradecimientos: Este trabajo se ha realizado en el marco del proyecto de CAICYT PB85-226. Dirección del autor: Facultad de Psicología. Universidad Autónoma de Madrid. 28049 Madrid. 


\section{INTRODUCCION}

El fenómeno de la mentira como elemento modulador de las relaciones sociales, ha sido objeto de atención por parte de pensadores claves en la constitución del pensamiento psicosocial moderno (Simmel, 1977; Pareto, 1946, citado en Lopreato, 1984, pág. 58-60).

Así también, Goffman (1959), en su modelo de "control de las impresiones" sanciona que la mentira preside todas las interacciones sociales, en la medida que las personas se vinculan en ejecuciones dramatizadas con el fin de controlar las impresiones que dan a los demás.

Desde una perspectiva experimental, el estudio de la mentira se ha desarrollado fundamentalmente en torno "al modelo teórico de filtraje», tal como Buck (1984) lo denomina. En sus trabajos en Comunicación no Verbal aplicados a la psicoterapia, donde se recogen influencias de la obra de Dal win (1872), Freud (1959) y el citado Goffman, Ekman y Friesen (1969) sitúan el foco de análisis del fenómeno en un ámbito más reducido y concreto que el de Goffman.

Las situaciones de mentira se distinguen de otros tipo de interacciones sociales porque emisor y receptor son conscientes de la posibilidad de aparición de mensajes falsos, la adopción de roles de mentiroso y detector durante la interacción y por la existencia de colaboración o antagonismo entre emisor y receptor; así también, los autores subrayan en este contexto, la importancia, de la capacidad diferencial de emitir mensajes de la cara y cuerpo.

En síntesis, el modelo de Ekman y Friesen (1969) propone que la emisión de la mentira va acompañada por expresiones de distinta naturaleza denominadas indicadores de mentira, los cuales transitan por distintos canales expresivos (faciales, corporales, verbales y paraverbales), constituyendo la detección de la mentira un proceso de captación, por parte del receptor, de los mencionados indicadores.

Otras formulaciones extraídas del modelo y relativamente apoyadas por la evidencia empírica (Ekman y Friesen, 1969, 1974; De Paulo y Fisher, 1981), son que las expresiones faciales son más controlables que el cuerpo, revelando (filtrando) con más dificultad que este último los indicadores de mentira. En relación con ello, la capacidad de control que el sujeto pueda ejercer sobre sí mismo durante la emisión de la mentira se revela como otra de las variables importantes en este contexto (Ekman y Friesen, op. cit. Elliot, 1979).

Estos desarrollos iniciales del modelo de filtraje, fijaron la atención sobre las características expresivas visuales (movimientos, gestos, etc.) que acompañan al mensaje fraudulento. Sin embargo, distintos trabajos empíricos llevados a cabo bajo los mismos'supuestos teóricos, han incidido sobre la presencia de determinados indicadores de carácter verbal y paraverbal en el momento de la emisión de mentiras.

Así, distintos investigadores, han trabajado sobre aspectos tales como el tono de voz (Scherer. y cols., 1985); latencias y longitudes de respuesta (Harrison y cols., 1978; Littlepage y Pinealut, 1981), expresiones negativas y errores sintácticos cometidos durante la emisión de mensajes falsos (ver Zuckerman y cols. 1981; Becerra, 1988, para una revisión).

Otro tipo de variables no deducidas del modelo, como motivación, sexo, 
edad, personalidad, etc., han sido estudiadas si bien los resultados obtenidos distan mucho de ser uniformes (De Paulo y cols., 1983; Feldman y cols. (1979); De Paulo y cols., 1979).

El objetivo de nuestro trabajo empírico, que se desarrolla en torno al modelo de filtraje, ha sido profundizar en el análisis de algunas de las variables implicadas en el fenómeno de la detección de la mentira, y que o bien presentan resultados contradictorios o no han sido tratadas con la extensión suficiente en la literatura revisada.

Tradicionalmente, el mensaje que transmite una mentira ha sido tratado de forma dicotómica (Ekman, Friesen, 1974; Zuckerman y cols. 1979) esto es, se ha considerado que el mensaje era verdadero (coincide con la realidad) o falso (no coincidente con la realidad) en sentido absoluto. Sin embargo, dado que el modelo de filtraje postula que la emisión de indicadores está determinada por procesos cognitivos, emocionales y/o activadores en general (Zuckerman y cols., 1985, para una revisión) que se disparan paralelamente a la emisión de la mentira, y cuyo fracaso para controlarlos permite su expresión externa, se ha considerado la posibilidad de que dichos procesos variasen en alguna dimensión según el tipo de mentira que se esté emitiendo.

Concretamente se ha pensado en una división de la mentira, según que su contenido fuera deseable o positivo para el sujeto que miente, a la que hemos llamado mentira deseable, o bien que el contenido fuese abiertamente indeseable para el sujeto que lo emite, observándose a continuación si se producen diferencias en la detección según la clase de mentiras.

Por otra parte, Buck (1984) recapitulando y extendiendo las propuestas del modelo de filtraje, hace hincapié en la necesidad de distinguir las mentiras emocionales de las no emocionales. La distinción lleva a considerar la importancia del contenido o tema de las mentiras en relación con su detección. Según esto, y en relación con la filtración de indicadores, ditintos temas tendrán distintas implicaciones emocionales para el sujeto que miente, lo cual supondrá una distinta activación emotiva en el mentiroso, que se corresponderá con una mayor o menor filtración de indicadores, y por tanto con una mayor o menor detección. En el presente trabajo se explora esta posibilidad.

Dado que el modelo de filtraje puede implicar diferencias individuales en el control de los indicadores de mentira y sus procesos subyacentes, la posibilidad de que unos individuos sean más detectables en sus mentiras que otros, será también explorada en este trabajo.

Finalmente y en razón de la existencia de resultados no concluyentes en relación con la variable sexo (Zuckerman y cols., 1979; De Paulo y cols., 1981) la incidencia de dicha variable sobre la detección de la mentira, será también indagada.

\section{METODO}

\section{Planteamiento General}

El trabajo empírico consistió en el visionado por parte de los sujetos experimentales de cuatro cintas de vídeo donde dos hombres y dos mujeres respondian en distintas formas a una serie de preguntas sobre diversos 
temas. Tras la conclusión de cada respuesta, los sujetos experimentales calificaban como verdadera o falsa la respuesta escuchada. La confección del material de grabación, y la puesta en práctica del diseño resultaron de gran complejidad. A los interesados en el procedimiento les remitimos al trabajo de Becerra y Sánchez (1989), donde se detallan con precisión todos los aspectos relacionados con el mismo.

\section{Instrumentos}

1. Grabaciones de vídeo. Cuatro películas, una por cada emisor, conteniendo sus respuestas a preguntas de ocho temas distintos, cuatro de temas considerados no emocionales (barrio, afición, etc.) y otros cuatro de temas emocionales (distintos tipos de relaciones intersexuales). El total de las respuestas emitidas fue de 96,24 por emisor. Cada emisor respondía a la misma prengunta de tres formas diferentes: Con verdad (respuesta verdadera), con una mentira cuyo contenido no le importaría al sujeto que fuese verdad (respuesta deseable) y con mentira, cuyo enunciado el sujeto no querría nunca que fuese verdad.

2. Hojas de respuesta. Protocolos estandarizados donde los sujetos experimentales marcaban sus juicios respecto a cada una de las respuestas de los emisores que se les mostraba.

3. Sujetos. Sesenta personas ( 30 hombres y 30 mujeres) voluntarios, de edades comprendidas entre 20 y 25 años (moda $=23$ en hombres $/ 22$ en mujeres) todos estudiantes universitarios de tecer curso de la Facultad de Psicología de la Universidad Autónoma de Madrid, que fueron distribuidos en 3 grupos de 10 hombres y 10 mujeres cada uno, de acuerdo al diseño experimental.

4. Procedimiento. Con las 96 respuestas emitidas por los 4 sujetos emisores se construyeron tres combinaciones diferentes, aunque análogas estructuralmente, con las respuestas verdaderas, deseables y falsas que se asignaron aleatoriamente a los sujetos de la muestra según sexo $(10 \times 10 \times 10$ hombres) y $(10 \times 10 \times 10$ mujeres $)$ a medida que iban tomando parte en el experimento.

\section{DISEÑO}

La operativización de las variables independientes fue como sigue:

1) Variable «Sujeto Emisor» (E), con cuatro condiciones correspondientes a los cuatro sujetos utilizados en la construcción de las grabaciones en video-tape (E1, E2, E3, E4) agrupados en Varón (E2 y E4) y Mujer (E1 y E3).

2) Variable «Tema» $(\mathrm{T})$, que se correspondía con el «tema» o «tópico» al que hacía referencia cada una de las ocho preguntas a las que respondían los «sujetos-estímulo".

3) Variable "Tipo de Expresión" (E) que, tal como hemos indicado, se corresponde con las tres modalidades de respuesta "verdadera» (EV) «falsa-deseable» (ED) y "no deseable" (EF).

4) Variable "Sexo del receptor" (S) que se corresponde con el sexo biológico del sujeto experimental que emitía los juicios de verdad/falsedad. 
En conjunto estas variables independientes quedaban articuladas en un diseño $4 \times 8 \times 3 \times 2$.

En cuanto a la variable dependiente, evidentemente, lo que se recogia era las respuestas de los sujetos en términos de juicios «verdadero" (V) o "falso" (F). En definitiva, nuestro interés se centraba en si el sujeto "acertaba» (A) o cometía un error (E) al juzgar las expresiones de los sujetos emisores. Por tanto era esta última la operacionalización efectiva de la variable dependiente.

A título descriptivo se construyeron las tablas de frecuencia de aciertos (A) y errores (E) respecto del Tipo de Expresión (Tabla I) y el tema (Tabla II).

TABLA I

Frecuencias de Aciertos y Errores por tipo de Expresión

\begin{tabular}{llll}
\hline & A & E & $\Sigma$ \\
\hline EV & 404 & 236 & 640 \\
\hline ED & 290 & 349 & 639 \\
\hline EF & 339 & 302 & 641 \\
\hline$\Sigma$ & 1.033 & 887 & 1.920 \\
\hline
\end{tabular}

TABLA II

Frecuencias de Aciertos y Errores por Tema

\begin{tabular}{lll}
\hline & A & E \\
\hline T1 & 137 & 103 \\
\hline T2 & 139 & 101 \\
\hline T3 & 105 & 135 \\
\hline T4 & 132 & 108 \\
\hline T5 & 121 & 119 \\
\hline T6 & 137 & 103 \\
\hline T7 & 127 & 113 \\
\hline T8 & 135 & 105 \\
\hline
\end{tabular}

Una inspección visual de estas tablas muestra que las respuestas de los sujetos siguen una tónica homogénea y relativamente esperable. No aparecen sesgos muy marcados que serían «sospechosos» dado que, por construcción, asumíamos que la situación experimental debía resultar «equilibrada». 
Para el análisis de los datos brutos se utilizaron estadísticos de contraste que suministran información sobre el grado de dependencia o asociación entre las variables objeto de estudio. Concretamente se ha llevado a cabo un análisis secuencial mediante modelos lineales logarítmicos para mas de dos factores (7D; ANOVA 1 y 2 factores; 4F del BMDP/1986).

Los análisis llevados a cabo a propósito de la naturaleza de nuestras variables (nominales) y el diseño planteado exige una determinación de las relaciones existentes entre las variables manipuladas por nosotros y los aciertos y los errores.

En razón de ello se optó por estadísticos basados en el análisis de la asociación o independencia entre variables según las distribuciones de frecuencias de cada una de ellas. En concreto los datos se analizaron mediante modelos matemáticos lineales logarítmicos.

El modelo aquí utilizado se basa en los estadísticos $\mathrm{X}^{2}$ y $\mathrm{G}^{2}$ independencia. Los cálculos se realizan determinando la relevancia de todos los efectos posibles de cada una de las variables contempladas, tomadas de forma independiente (efectos principales), dos a dos (efectos de segundo orden), tres a tres (efectos de tercer orden), etc.

La presencia de valores significativos de $\mathrm{X}^{2}$ marginal y parcial sobre los correspondientes efectos analizados determina que dicho efecto sea considerado importante para el modelo de representación de los datos. La no presencia de significación en las pruebas de asociación parcial y marginal sugiere la no relevancia del efecto de cara al modelo. La aparición de significación en una sola de las pruebas, sugiere una menor relevancia del efecto y la necesidad de seguir analizando su aportación real al modelo.

Debe hacerse notar que la estrategia de comprobación de los modelos lineales logarítmicos consiste en comprobar el grado de ajuste entre la distribución de las frecuencias observadas y las frecuencias esperadas a partir de un modelo establecido que, para nuestro caso, es la distribución al azar.

El grado de ajuste entre las frecuencias esperadas y las observadas a partir de un modelo lineal logarítmico viene dado por un bajo valor del coeficiente, esto es: las frecuencias observadas poseen una cierta distribución semejante a la predicha por el modelo. En nuestro caso un valor bajo de $\mathrm{X}^{2}$ denota pues que la distribución de los valores se ajusta a una distribución al azar y un valor alto, lo contrario. bla III.

Los resultados obtenidos mediante estos análisis aparecen en la Ta-

\section{RESULTADOS}

Las conclusiones que se pueden extraer de los resultados obtenidos apuntan en primer lugar, que la variable Aciertos muestra diferencias significativas, entre las categorías de Aciertos y Errores, lo que sugiere el carácter no aleatorio de los juicios emitidos por los sujetos experimentales.

En cuanto a los efectos de segundo orden se puede comprobar como la variable Tema (T) y Tipo de Expresión (X) se relacionan con los aciertos, en mayor medida que con los errores, lo que, si bien de forma descriptiva, otorga ciertos apoyos a algunos de nuestros planteamientos. En este nivel, señalar el hecho de que la variable Sujeto Emisor y Sexo no guardan mayor relación con los aciertos que con los errores. 


\section{TABLA III}

Modelo lineal logaritmico

(Efectos de las variables "Sexo* (S), tipo de *Expresión * $(X)$, *Tema* (T), «Sujeto-Emisor" (E) $y$ *Aciertos* $(A)$; en subrayado los estadisticos significativos)

\begin{tabular}{|c|c|c|c|c|c|}
\hline \multirow{2}{*}{ Efecto } & \multirow{2}{*}{$\underset{\mathbf{X}^{2}}{\text { G.L. }}$} & \multicolumn{2}{|c|}{ Asociación parcial } & \multicolumn{2}{|c|}{ Asociación marginal } \\
\hline & & $\mathbf{X}^{2}$ & Probab. & $\mathbf{X}^{2}$ & Probab. \\
\hline $\begin{array}{l}A \\
S \\
X \\
T \\
E\end{array}$ & $\begin{array}{l}1 \\
1 \\
2 \\
7 \\
3\end{array}$ & $\begin{array}{r}10.10 \\
0.00 \\
0.00 \\
0.00 \\
0.00\end{array}$ & $\begin{array}{l}0.0015 \\
1.0000 \\
0.9987 \\
1.0000 \\
1.0000\end{array}$ & & \\
\hline $\begin{array}{l}\text { AS } \\
\text { A X } \\
\text { A T } \\
\text { A E } \\
\text { S X } \\
\text { S T } \\
\text { S E } \\
\text { X T } \\
X \text { E } \\
\text { T E }\end{array}$ & $\begin{array}{r}1 \\
2 \\
7 \\
3 \\
2 \\
7 \\
3 \\
14 \\
6 \\
21\end{array}$ & $\begin{array}{r}0.16 \\
37.56 \\
14.20 \\
1.91 \\
0.01 \\
0.00 \\
0.00 \\
0.26 \\
0.05 \\
0.01\end{array}$ & $\begin{array}{l}0.6888 \\
0.0000 \\
0.0478 \\
0.5918 \\
0.9961 \\
1.000 \\
1.000 \\
1.000 \\
1.000 \\
1.000\end{array}$ & $\begin{array}{r}0.15 \\
37.28 \\
13.97 \\
1.85 \\
0.00 \\
0.00 \\
0.00 \\
0.02 \\
0.01 \\
0.00\end{array}$ & $\begin{array}{l}0.6945 \\
0.0000 \\
0.0522 \\
0.6046 \\
0.9985 \\
1.0000 \\
1.0000 \\
1.0000 \\
1.0000 \\
1.0000\end{array}$ \\
\hline $\begin{array}{l}\text { A S X } \\
\text { A S T } \\
\text { A S E } \\
\text { A X T } \\
\text { A X E } \\
\text { A T E } \\
\text { S X T } \\
\text { S X E } \\
\text { S T E } \\
\text { E T E }\end{array}$ & $\begin{array}{r}2 \\
7 \\
3 \\
14 \\
6 \\
21 \\
42 \\
6 \\
21 \\
42\end{array}$ & $\begin{array}{r}1.77 \\
6.42 \\
1.58 \\
48.52 \\
65.19 \\
62.82 \\
0.39 \\
0.13 \\
0.30 \\
4.85\end{array}$ & $\begin{array}{l}0.4125 \\
0.4922 \\
0.6635 \\
0.0000 \\
0.0000 \\
0.0000 \\
1.0000 \\
1.0000 \\
1.0000 \\
1.0000\end{array}$ & $\begin{array}{r}1.66 \\
5.83 \\
1.39 \\
46.77 \\
61.57 \\
58.76 \\
0.02 \\
0.01 \\
0.00 \\
0.06\end{array}$ & $\begin{array}{l}0.4365 \\
0.5602 \\
0.7084 \\
0.0000 \\
0.0000 \\
0.0000 \\
1.0000 \\
1.0000 \\
1.0000 \\
1.0000\end{array}$ \\
\hline $\begin{array}{l}\text { A S X T } \\
\text { A S E T } \\
\text { A S X E } \\
\text { A X T E } \\
\text { SX T E }\end{array}$ & $\begin{array}{r}14 \\
6 \\
21 \\
42 \\
42\end{array}$ & $\begin{array}{r}19.00 \\
7.02 \\
25.17 \\
127.09 \\
134.57\end{array}$ & $\begin{array}{l}0.1648 \\
0.3193 \\
0.2400 \\
0.0000 \\
1.0000\end{array}$ & $\begin{array}{r}16.70 \\
6.03 \\
22.54 \\
125.52 \\
130.06\end{array}$ & $\begin{array}{l}0.2724 \\
0.4197 \\
0.3687 \\
0.0000 \\
1.0000\end{array}$ \\
\hline A S X T E & 42 & 46.99 & 0.2755 & & \\
\hline
\end{tabular}

El análisis de los efectos de segundo orden y tercer orden, presentan nuevas dimensiones del fenómeno de la detección de la mentira de carácter más complejo que el mencionado anteriormente por nosotros.

Así, la variable Sujeto Emisor que no aparecía como significativa considerada aisladamente, en asociación con el resto de las variables si lo es, lo que nos lleva a pensar que el fenómeno de la detección de la mentira, es de naturaleza más global y compleja que la simple expresión y detección de indicadores. Impresión ésta, que se confirma a través de los análisis de los efectos de cuarto orden, en donde la variable aciertos se relaciona con la totalidad de las variables seleccionadas salvo la variable sexo que no presenta en ningún caso efectos significativos.

Si bien estas asociaciones implican necesariamente un cierto reconocimiento de los mensajes falsos, pareció aconsejable profundizar en el análisis de los datos, contrastando los juicios que los sujetos experimentales emitieron, exclusivamente, sobre las respuestas falsas y deseables, para lo cual se volvieron a utilizar los programas estadísticos descritos. 
En este sentido y en relación con las expresiones falsas puede afirmarse que los sujetos experimentales, tanto hombres como mujeres, considerados globalmente aciertan más en las expresiones referidas a los temas 5-8 (192 aciertos, 129 errores), los considerados por nosotros como emocionales, que en las pertenecientes a los temas 1-4 (147 aciertos, 175 errores) considerados no emocionales $\left(\mathrm{X}^{2}=12.40, \mathrm{p}<0.01\right)$, lo cual es congruente con nuestra hipótesis de que determinados temas suponen mayor activación de los sujetos-emisores y por lo tanto una mayor posibilidad de filtración de indicadores.

En relación con la asociación Aciertos, Tipo de expresión, Sujetos Emisores, cabe destacar que los mensajes falsos (expresiones falsas) de uno de los emisores, el denominado E4 (104 aciertos, 56 errores), son significativamente más detectadas por los sujetos experimentales que para el resto de los emisores $\left(\mathrm{X}^{2}=23.3 ; \mathrm{p}<0.01\right)$, resultado éste que tentativamente puede considerarse como evidencia de diferente expresividad o de control de las expresiones por parte de los sujetos emisores, algo coincidente con nuestras hipótesis de partida.

Con todo, hay que hacer constar que la detección de las expresiones falsas presenta un patrón de asociación entre variables semejante al expuesto en la Tabla 3, de lo que puede deducirse igualmente que la detección de expresiones falsas es en última instancia función de una interconexión entre las variables Tema y Sujeto-Emisor $\left(\mathrm{X}^{2}=77.16 ; \mathrm{p}<0.01\right)$.

El mismo análisis llevado a cabo en relación con las expresiones deseables, muestra diferencias interesantes en relación con las expresiones falsas.

En general, los sujetos experimentales cometen significativamente más errores al juzgar las expresiones deseables que las falsas (290 aciertos, 349 errores vs. 339 aciertos, 302 errores) $\left(\mathrm{X}^{2}=7.19 ; \mathrm{p}<0.01\right)$, pudiendo considerarse estos resultados como una primera evidencia de que existen diferencias entre los tipos de mentira, que tienen implicaciones ulteriores sobre la conducta, toda vez que para el sujeto-emisor que miente, las expresiones deseables no guardan el mismo significado que las expresiones falsas, con las posibles diferencias en activación emocional y la correspondiente filtración.

Considerando la asociación conjunta de todas las variables implicadas obtenemos que el emisor 4 es más detectado en las expresiones deseables de los temas emocionales cuando se le compara con los emisores 1 y 3 con $\mathrm{X}^{2}=4.24, \mathrm{p}<0.05 ; \mathrm{X}^{2}=8.88$. $\mathrm{p}<0.01 ;$ no apareciendo diferencias respecto al emisor 2 .

\section{DISCUSION}

Como se recordará el objetivo de este estudio era analizar la influencia de determinadas variables (tema del mensaje, tipo de expresión, sujeto emisor del mensaje, sexo del receptor que evalúa el mensaje y aciertos) en la detección de la mentira expresada.

Tras un primer análisis de los datos, comprobamos que los aciertos eran más numerosos que los errores, evidenciando que los sujetos experimentales llevan a cabo con éxito la tarea encomendada. Sin embargo, los aciertos no están vinculados en particular con ninguna de las variables manipula- 
das, sino que son fruto de algún tipo de interacción compleja entre ellas, por lo que entramos en un análisis más pormenorizado de las variables que nos interesaba analizar.

Así, encontramos una primera interacción significativa entre tema y acierto, que apunta que el tema sobre el que se construye la expresión del mensaje influye tanto en la presentación que el emisor hace de éste, como en la interpretación que de dicho mensaje hará el receptor, y en este sentido se puede comprobar que la consideración de temas emocionales y no emocionales responde a las expectativas que generaron el planteamiento de nuestra hipótesis, los temas emocionales generan una presentación diferencial, a los temas no emocionales, que es detectada por los sujetos a quienes va dirigido el mensaje.

En lo referido al sujeto emisor, los resultados del presente trabajo apoyarían estas consideraciones, ya que la mentira (los mensajes falsos) es detectada en todos los sujetos emisores, pero lo es mejor en unos que en otros, y así el que nosotros hemos llamado emisor 4, es mejor detectado en sus mentiras que el resto de los sujetos emisores. Respecto a este sujeto, podríamos pensar que tiene un menos control sobre las conductas verbales y no verbales que acompañan a los mensajes falsos, de tal forma que «filtra» más, o sea que deja entrever mejor, a quienes le observan, que está mintiendo.

Por lo que respecta a las variables tipo de expresión aparecen diferencias entre las formas posibles en que pueden aparecer los mensajes falsos. Como hemos descrito en el apartado de resultados, hay mensajes falsos más fáciles de identificar que otros, por parte de sujetos no expertos, y en nuestro caso los mensajes que eran mentiras, pero deseables, fueron peor reconocidos que los mensajes auténticamente falsos, con contenidos indeseables.

Una explicación de esta diferencia respecto a las variables tema y sujeto emisor, apuntaría a que el emisor no se sentiría «tan perturbado» al emitir mensajes deseables como al emitir mensajes completamente falsos, lo que evitaría un aumento en los niveles de activación, no produciéndose cambios importantes en la representación que se le pide, porque quizás el emisor, no considera que esté mintiendo y lo que expresa mediante una expresión deseable es algo que puede estar más próximo a una consideración verdadera, que a una consideración falsa, teniendo menos dificultades para aparecer convincente ante los demás.

Por contra, y en relación con la emisión de mensajes falsos, la mayor activación «sufrida» por el sujeto emisor que miente, redundaría, de acuerdo con la teoria, en una mayor presencia de indicadores de engaño, que sería detectada por los sujetos emisores.

Antes de finalizar, queremos apuntar brevemente que el sexo de los sujetos receptores no ha tenido la relevancia que presuponíamos al plantear este trabajo, no apareciendo diferencias en la detección, traducida en número de aciertos, que hombres y mujeres hacen de los mensajes falsos.

Tras la consideración de todo lo expuesto, nuestros esfuerzos dentro de este campo, se orientarán a conseguir una mayor definición de las variables que influyen en la presentación que los emisores hacen de los mensajes falsos, y que en determinadas condiciones alertan al receptor de que está inmerso en un proceso de comunicación fraudulenta. En este sentido, y apoyándonos en nuestros datos empíricos (Becerra, Sánchez y Carrera, 1989), 
consideramos interesante seguir explorando la existencia de un patrón general de configuración, idiosincrásico para cada sujeto, que variaría cualitativamente en la emisión de mensajes verdaderos y falsos, siendo percibidas tales variaciones por los receptores de los mensajes.

\section{Referencias}

Becerra, A. (1988). aLa detección de la mentira expresada». Tesis doctoral. Facultad de Psicología, Universidad Autónoma de Madrid.

BeCERRA, A. y SÁNCHEZ, F. (1989). "Procedimiento experimental para la indagación del fenómeno de la detección de la mentira». Evaluación Psicológica/Psychological Assessment vol. 5 (1), 55-68.

Becerra, A.; SÁNChez, F. y Carrera, P. (1989). «Indicadores aislados vs. patrón general expresivo en la detección de la mentira». (En Prensa).

BUCK, Ross (1984). The communnication of Emotion, Guilford Press, Nueva York.

DARWIN, C. (1872). La expresión de las emociones en el hombre y los animales. Trad. Alianza, Madrid, 1984.

De Paulo, B. M. y Rosenthal, R. (1979). "Telling Lies». Journal of Personality and Social Psychology, 37, 1713-1722.

De PAUlo, B. M. y FiShER, J. P. (1981). Too tuned-out to take: The role of nonverbal sensitivity in help seeking. Personality and Social Psychology Bulletin, 7.

De Paulo, B. M.; LAINER, K. y Davis, T. (1983). "Detecting the Deceit of the Motivated Liar". Journal of Personality and Social Psychology, 45 (5), 1096-1103.

EKMAN, P. y FRIESEN, W. V. (1969). Nonverbal leakage and clues to deception. Psychiatry, 32, 88-106.

EKMAN, P. y FRIESEN, W. V. (1974). «Detecting Deception from the Body or Face». Journal of Personality and Social Psychology, 29 (3), 288-298.

Elliot, G. C. (1979). "Some Effects to Deception an Level of Self-Monitoring on Planning and Reactin to a Self-Presentation». Joumal of Personality and Social Psychology, 37 (8), 1282-1292.

Feldman, R. S.; Jenkins, L. y POPOOLA, O. (1979), "Detection of deception in adults and children via facial expressions». Child Development, s0, 350-55.

FREUD, S. (1959). "Fragment of an Analysis of a Case of Hysteria» (1905). Collected Papers, 3. Basic Book.

Goffman, E. (1959). La presentación de la persona en la vida cotidiana (Trad. cast. Amorrortu-Murguia, 1987).

Harrison, A.; Hwalek, M.; Raney, D. y FriTZ, J. G. (1978). «Cues to Detection in an Interview Situation». Social Psychology, 41 (2), 156-161.

Littlepage, G. E. y Pineault, M. A. (1981). "Detection of thuthful and Deceptive Interpersonal Communications Across Information Transmission modes». Journal of Social Psychology, 114, 57-68.

LOPREATO, J. (1984). Human nature and Biocultural Evolution. Allen and Unwin. Boston.

SCHERER, K. R.; FELDSTEIN, S.; BOND, R. N. y RoSENTHAL, R. (1985). "Vocal cues to deception: a comparative channel approach». Journal of Psycholinguistic Research, 14 (4), 409-25.

Simmel, G. (1977). Sociología 1 (Estudios sobre la forma de socialización). Revista de Occidente. Madrid.

Zuckerman, M.; Defrank, R. S.; Hall, J. A.; Larrance, D. T. y Rosenthal, R. (1979). "Facial and vocal cues of deception and honesty". Joumal of Experimental Social Psycho$\log y, 15,378-96$.

Zuckerman, M.; DePaulo, B. M. y Rosenthal, R. (1981). Verbal and Nonverbal Communication of Deception. En L. Berkovitz (comp.). Advances in Experimental Social Psychology, 14, Academic Press, Nueva York.

ZuCKERMAN, M. y DRIVER, R. E. (1985). Telling Lies: Verbal and Nonverbal Correlates of Deception. Cap. 5 en A. W. Siegman, S. Feldstein. Multichannel Integrations of Nonverbal Behavior. L.E.A., Nueva Jersey. 